Article type : Articles

${ }^{1}$ School of Ecosystem and Forest Sciences, University of Melbourne, 4 Water Street, Creswick,

18 Victoria, Australia

Authors: Holly Sitters ${ }^{1} *$

Running head: Generating precise conservation targets

\title{
Survey design for precise fire management
}

\section{conservation targets}

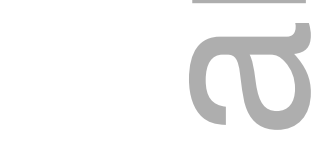

Julian Di Stefano ${ }^{1}$

${ }^{2}$ GHD, Level 8, 180 Lonsdale Street, Melbourne, Victoria, Australia

This is the author manuscript accepted for publication and has undergone full peer review but has not been through the copyediting, typesetting, pagination and proofreading process, which may lead to differences between this version and the Version of Record. Please cite this article as doi: $\underline{10.1002 / \text { eap. } 1624}$

This article is protected by copyright. All rights reserved 
$21 *$ Corresponding author E-mail: holly.sitters@unimelb.edu.au
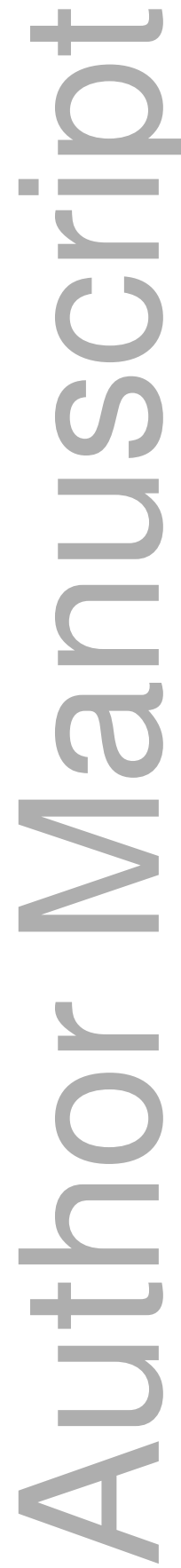

This article is protected by copyright. All rights reserved 


\section{Abstract}

23 Common goals of ecological fire management are to sustain biodiversity and minimize

24 extinction risk. A novel approach to achieving these goals determines the relative proportions of

25 vegetation growth stages (equivalent to successional stages, which are categorical

26 representations of time since fire) that maximize a biodiversity index. The method combines

27 data describing species abundances in each growth stage with numerical optimization to define

28 an optimal growth-stage structure which provides a conservation-based operational target for

29 managers. However, conservation targets derived from growth-stage optimization are likely to

30 depend critically on choices regarding input data. There is growing interest in use of growth-

31 stage optimization as a basis for fire management, thus understanding of how input data

32 influence the outputs is crucial. Simulated datasets provide a flexible platform for systematically

33 varying aspects of survey design and species inclusions. We used artificial data with known

34 properties, and a case-study dataset from southeastern Australia, to examine the influence of (i)

35 survey design (total number of sites, and their distribution among growth stages) and (ii) species

36 inclusions (total number of species and their level of specialization) on the precision of

37 conservation targets. Based on our findings, we recommend that survey designs for precise

38 estimates would ideally involve at least 80 sites, and include at least 80 species. Greater

39 numbers of sites and species will yield increasingly reliable results, but fewer might be sufficient

40 in some circumstances. An even distribution of sites among growth stages was less important

41 than the total number of sites, and omission of species is unlikely to have a major influence on

42 results as long as several species specialize on each growth stage. We highlight the importance

43 of examining the responses of individual species to growth stage before feeding survey data into

44 the growth-stage optimization black box, and advocate use of a resampling procedure to

45 determine the precision of results. Collectively, our findings form a reproducible guide to

46 designing ecological surveys that yield precise conservation targets through growth-stage

47 optimization, and ultimately help sustain biodiversity in fire-prone systems.

48 Key words biodiversity conservation, ecological monitoring, fire management, land

49 management, prescribed burning, sampling design, sampling framework, simulation

This article is protected by copyright. All rights reserved 
51 Fire is a natural source of disturbance that drives ecosystem structure and function (Bond and

52 Keeley 2005). It is also used as a management tool in flammable biomes of Africa, Australia,

53 North America and Europe, both to reduce the risk of wildfire and achieve conservation

54 objectives (Penman et al. 2011; Stephens et al. 2012; Fernandes et al. 2013). Common aims of

55 ecological fire management are to sustain biodiversity and minimize extinction risk, but it is

56 rarely clear how best to apply fire to enhance biodiversity.

57 Ecological fire management is often guided by the notion that variable fire regimes promote

58 biodiversity (Bradstock et al. 2005). Vegetation growth stages (equivalent to successional

59 stages, which are categorical representations of time since fire) can be used as measures of fire-

60 mediated landscape variation. In theory, different species' resource requirements are met by

61 different growth stages, and the responses of individual species to growth stage are reflected in

62 shifts in community composition along successional gradients (Fox 1982; Pons and Clavero

63 2010). A heterogeneous landscape composed of several growth stages is therefore expected to

64 support more species than a homogeneous landscape (Martin and Sapsis 1992; Parr and

65 Andersen 2006). Some studies have identified positive associations between species diversity

66 and growth stage diversity (Fuhlendorf et al. 2010; Maravalhas and Vasconcelos 2014; Sitters et

67 al. 2014a), while others have highlighted the disproportionate importance of particular growth

68 stages (Davies et al. 2012; Kelly et al. 2012; Taylor et al. 2012; Farnsworth et al. 2014; Robinson

69 et al. 2014; Burgess and Maron 2016; Doherty et al. 2017).

70 Knowledge gaps regarding appropriate levels of growth-stage diversity can hamper the use of

71 planned fire as a conservation tool (Driscoll et al. 2010). However, a new approach to

72 multispecies conservation defines the relative proportions of different growth stages that

73 maximize a biodiversity index (McCarthy 2012; Di Stefano et al. 2013; Kelly et al. 2015). The

74 method combines data describing species abundances in different growth stages with numerical

75 optimization to determine an optimal growth-stage structure, providing a conservation-based

76 operational target for fire managers. Growth-stage optimization has recently been incorporated

77 into fire-management policy in southeastern Australia because it has a strong theoretical basis

78 (McCarthy et al. 2014), input data can be obtained using standard ecological survey techniques, 
and it provides a unified framework for addressing conservation goals associated with multiple

80 species (DELWP 2015).

81 Growth-stage optimization presents a powerful means of linking real-world operational objectives with desirable conservation outcomes. For a particular set of input data the process

83 generates two outputs; an optimal growth-stage structure and the value of a biodiversity index

84 that is theoretically at its maximum - hereafter we refer to these outputs as "conservation targets" and "optimization results". Both outputs are useful for fire-management planning; the optimal growth-stage structure can be used as an operational target while the maximised value of

87 the biodiversity index can be used as a benchmark to assess the ecological effects of different

88 fire-management scenarios. However, the optimization results for a given ecosystem are likely to depend critically on choices regarding input data, including ecological survey design and the species included in the analysis (Di Stefano et al. 2013; Giljohann et al. 2015; Kelly et al. 2015). For example, species that specialize on particular growth stages should have a greater influence on the optimal growth-stage structure than generalist species, but the sensitivity of results to the inclusion or exclusion of specialists has not been systematically tested. There is growing use of growth-stage optimization as a basis for ecological fire management (Di Stefano et al. 2013; DELWP 2015; Giljohann et al. 2015; Kelly et al. 2015), thus better understanding of the influence of survey choices on optimization results is crucial to ensure that managers are able to

97 make sound investment decisions.

98 Simulation provides a flexible means of systematically varying aspects of ecological data sets 99 (Schweiger et al. 2016). In this paper we use artificial data with known properties to test the 100 influence of (i) survey design (total number of sites, and the distribution of sites among growth 101 stages) and (ii) species inclusions (total number of species and the influence of removing growth102 stage specialists) on optimization results. To enhance our ability to generalize inferences to 103 different ecosystems, we created two artificial communities, each containing 100 species and 104 differing in the proportion of species that responded to time since fire. Community A 105 represented systems in which the majority of species respond to time since fire, such as faunal 106 communities of southwestern USA forests (e.g. Kalies et al. 2010). In Community B fewer 107 species responded to time, and older vegetation was of disproportionate importance, as is the 108 case in some southeastern Australian faunal communities (Di Stefano et al. 2013; Kelly et al. 
2015). To ensure our simulation study produced realistic results, we also applied our species-

110 inclusion tests to empirical data collected in southeast Australian eucalypt forest. Our results

111 provide a reproducible guide to designing ecological surveys that generate precise conservation

112 targets through growth stage optimization, and improve its utility as a conservation tool.

\section{Methods}

114 The growth-stage optimization procedure involves the following steps (McCarthy 2012; Di

115 Stefano et al. 2013; Kelly et al. 2015) (1) biodiversity surveys at sites of different time since fire,

116 (2) development of statistical models relating individual species to time since fire, (3) converting

117 time since fire to growth-stage categories, and splitting model predictions by growth stage, (4)

118 using numerical optimization to determine the proportional coverage of each growth stage that

119 maximizes a biodiversity index. We sought to follow this process as closely as possible using

120 simulated data, ensuring that the characteristics of our artificial data represented typical faunal

121 community datasets.

\section{Artificial community datasets}

123 We used three response shapes (no response as a null model, linear response, and unimodal

124 response) to capture a diversity of species' responses to time since fire (Fig. 1). We scaled time

125 since fire $(x)$ from $0-100$ and species abundances $(y)$ from $0-1$. We generated a response

126 function for each species (Table 1, Data S1) by randomly selecting response-shape parameters

127 within a range defined using empirical data (Swan et al. n.d.; Sitters et al. 2014b), ensuring that

128 the slopes of relationships between abundance and time since fire were realistic. Our two

129 communities differed in the proportion of species that responded to time since fire;

130 approximately two thirds of species in Community A responded to time since fire, half of which

131 responded positively and half negatively. In Community B only one third of species responded

132 to time since fire, and most species preferred late-successional vegetation. Response functions

133 corresponding to all species in Communities A and B are provided in Data S1, and summarized

134 in Table 1. We used each species' response function to generate 50 abundance values per

135 growth stage. For each community, this yielded a data matrix comprising 50 observations per

136 species $(n=100)$ per growth stage $(n=4)$, which formed input data for all subsequent analyses, 
137 and represented a "best-case" survey design scenario which is probably rarely feasible in real-

138 world contexts. Our four growth stages reflect other optimization studies (Di Stefano et al. 2013;

139 Giljohann et al. 2015; Kelly et al. 2015; Hale et al. 2016), which have defined three or four

140 categories representing major stages in post-fire vegetation growth and development (Cheal

141 2010) (Fig. 1). We did not systematically vary survey effectiveness or species' detection

142 probabilities, and instead assumed that individuals were detected when present. Artificial data

143 were generated in the R statistical environment ( $\mathrm{R}$ Core Team 2016) using custom functions

144 modified from Schweiger et al. (2016) and the data manipulation packages plyr (Wickham

145 2011), dplyr (Wickham and Francois 2015) and reshape2 (Wickham 2007).

146 Growth-stage optimization

147 We used numerical optimization to determine the proportions of each growth stage that

148 maximized a biodiversity index, which was the geometric mean of species' abundances ( $\mathrm{Di}$

149 Stefano et al. 2013; Giljohann et al. 2015; Kelly et al. 2015). The geometric mean underpins

150 several large-scale biodiversity-monitoring initiatives because of its beneficial mathematical

151 properties (e.g. the Living Planet Index, Loh et al. 2005). For example, small differences in the

152 abundance of uncommon species among growth stages represent large relative differences, and

153 have a greater influence on the geometric mean than more common species that may show larger

154 absolute differences in abundance (Buckland et al. 2005, 2011). Further, the geometric mean

155 reflects parallel shifts in the absolute abundance of multiple species; in contrast, popular

156 diversity indices such as Shannon's and Simpson's remain constant when relative abundances

157 are unchanged (Buckland et al. 2005). The optimal growth-stage structure and biodiversity

158 index were calculated using local gradient-based optimization (the NLOPT_LD_MMA

159 algorithm; Svanberg 2002) in the R package nloptr (Johnson 2016), subject to the constraint that

160 the growth-stage proportions sum to 1 (code is provided in Data S2).

161 We tested the influence of sampling design and species inclusions on: (1) the precision of the

162 biodiversity index, (2) the precision of the optimal growth-stage structure, and (3) the shape of

163 the optimal growth-stage structure. We did this by subsampling our "best-case" artificial

164 datasets under various scenarios, and conducting growth-stage optimization on each subsampled

165 dataset (Fig. 2, Data S2). We used a resampling procedure to generate measures of precision; 
166 species abundance values in each growth stage were resampled with replacement, the mean

167 abundance per species per growth stage was calculated, and the process was repeated 1000 times.

168 Growth-stage optimization was conducted on each resampled dataset, and the mean biodiversity

169 index and optimal growth-stage structure were quantified. We measured the precision of the

170 biodiversity index by calculating the standard deviation of values associated with the 1000

171 iterations. To yield a single measure of precision corresponding to the optimal growth-stage

172 structure, we calculated the mean of the four standard deviations corresponding to each growth

173 stage. The workflow is illustrated in Fig. 2 and code is available in Data S2; resampling was

174 undertaken in $\mathrm{R}$ using the base package and plyr (Wickham 2011; R Core Team 2016).

\section{Survey-design scenarios}

176 We began by examining the influence of two aspects of survey design on the biodiversity index

177 and optimal growth-stage structure: the total number of sites, and the distribution of sites among

178 growth stages. We did this by subsampling the best-case artificial community datasets under

179 various scenarios.

180 The least exhaustive survey-design scenario we considered was 5 sites per growth stage, and we

181 systematically increased the number of sites per growth stage from 10-50 in increments of 10,

182 such that the maximum number of sites considered was the best-case scenario of 200 (50 sites $\times$

1834 growth stages). We were also interested in the extent to which the distribution of sites among

184 growth stages influenced the biodiversity index and optimal growth-stage structure. We

185 therefore limited the number of sites in each growth stage in turn to $10 \%$ of the total number of

186 sites, and divided the remaining sites evenly among the other growth stages. In total, we

187 examined 30 survey-design scenarios (Appendix S1: Table S1; six scenarios where the total

188 number of sites varied $(20,40,80,120,160,200) \times$ five scenarios where the distribution of sites

189 among growth stages varied (evenly distributed among growth stages; sites in each of the four

190 growth stages limited to $10 \%$ of the total)).

\section{Species-inclusion scenarios}

192 To examine the influence of species inclusions on optimization results we systematically varied

193 the total number of species included in the optimization routine, while keeping the number of 
194 sites constant at 50 per growth stage (i.e. the best-case survey-design scenario). We began by

195 including 10 species, and increased the number of species in increments of 10 to a maximum of

196 90. This required a modification to the resampling procedure described above; 100 subsets of $n$

197 species were randomly selected from the full species pool, and resampling was carried out as

198 before, to generate 100 resampled datasets per subset. Growth-stage optimization was carried

199 out on the resulting 10,000 resampled datasets. The precision and shape of optimization results

200 were derived from the 10,000 iterations.

201 Species that specialize on particular growth stages should have a greater influence on

202 optimization results than generalist species. To establish the influence of growth-stage

203 specialization on optimization results, we used the standardised measure $\left(B_{\mathrm{A}}\right)$ of Levins' index

204 of niche breadth $(B)$ to rank species (Levins 1968):

205

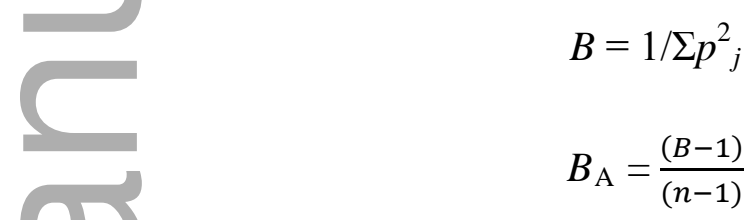

207 Where $p_{j}=$ proportion of the species' total abundance in growth stage $j$; and $n=$ the total number 208 of growth stages. This produces a single value per species ranging from 0 (if a species occurs in 209 only one growth stage) to 1 (if a species is equally abundant in all growth stages). We selected

210 species that had a Levins index $<0.5$ ( $n=27$ in Community A, $n=13$ in Community B), and

211 used their mean abundance per growth stage to determine the category on which they

212 specialized.

213 Next, we excluded a species that specialized on each growth stage in turn, and conducted

214 growth-stage optimization using the remaining species. We considered a total of 45 scenarios

215 (Appendix S1: Table S2; nine scenarios where the total number of species varied $\times$ five scenarios

216 where specialist species were excluded (including a scenario where no species were excluded)).

217 Preliminary analysis showed that exclusion of additional specialist species had negligible

218 influence on optimization results provided there were more than two specialists per growth stage.

219 The importance of the number of specialists per growth stage is captured in our 45 scenarios

220 because we limited the number of early-growth-stage specialists to two in Community A and one

221 in Community B. Again, we tested all scenarios on Communities A and B separately, and 
222 examined the precision of the biodiversity index, the precision of the optimal growth-stage

223 structure, and the shape of the optimal growth-stage structure.

\section{Testing species-inclusion scenarios on case-study data}

225 Finally, we sought to confirm that patterns evident in optimization results derived from artificial 226 data were reflected in those derived from empirical data, so we repeated our species-inclusion 227 tests using a case-study dataset collected in a 15,000-ha region of the Otway Ranges,

228 southeastern Australia. We did not test survey-design scenarios using this dataset because the 229 number of sites per growth stage was limited. Testing the species-inclusion scenarios involved

230 (1) surveys of ground-dwelling mammals, birds and vascular plants at sites of different time 231 since fire, (2) development of statistical models relating individual species to time since fire, (3) 232 splitting model predictions by growth stage, and (4) applying growth-stage optimization under 233 different species-inclusion scenarios.

234 Our study area was dominated by the forby forest Ecological Vegetation Division (EVD; Cheal 235 2010) and characterized by steep terrain (70-350 $\mathrm{m}$ above sea level) and a mild climate (mean 236 annual minimum and maximum temperatures, 10.5 and $18.2^{\circ} \mathrm{C}$; mean annual rainfall, $1229 \mathrm{~mm}$ )

237 (Bureau of Meteorology 2016). Vegetation was tall-open eucalypt forest supporting Eucalyptus 238 cypellocarpa, E. globulus, E. obliqua and E. radiata, and featuring a diverse ground layer of 239 forbs and grasses (Cheal 2010). The midstory shrub layer ranges from sparse to dense, and 240 canopy trees reach $50-60 \mathrm{~m}$ in height. Forby forest is flammable during the warmer months, and 241 growth stages are defined as: recently burnt (0-3 years since fire), early (4-10 years), middle (1124240 years), late (>40 years) (Cheal 2010; Cohn et al. 2015).

243 We used standard field methods to survey ground-dwelling mammals, diurnal birds and vascular 244 plants at 47 sites as part of a larger study examining relationships between fire and biodiversity 245 (see Sitters et al. 2014a for a detailed description of the study design). The spread of sites along 246 the time-since-fire gradient, and the corresponding number of sites per growth stage, reflected 247 the spatial coverage of each category at the time of field surveys (10 sites were in the recently248 burnt growth stage, 5 early, 11 middle, 21 late). Sites consisted of a 100-m transect positioned 249 along a random bearing, and all were surveyed in both 2010 and 2011. Small mammals (Rattus 250 spp. and Antechinus spp.) were surveyed at each site with aluminium box traps, and larger 
ground-dwelling mammals were surveyed with camera traps (see Swan et al. (2015) for details).

252 Diurnal birds were surveyed using 20-minute point counts undertaken at dawn and dusk (Sitters

253 et al. 2014a), and vascular plants were surveyed in 2- $\mathrm{m}^{2}$ quadrats placed at 3-m intervals along

254 transects (33 quadrats per site). Fauna are often the focus of growth-stage optimization (Kelly et

255 al. 2015; Hale et al. 2016), and our artificial communities were constructed to represent typical

256 faunal datasets. However, data from vertebrates and vascular plants have been used in

257 combination (Di Stefano et al. 2013), and here we use both flora and fauna data to generate a

258 large enough species pool to allow us to test the species-inclusion scenarios. Our case-study

259 dataset contained 107 species; 6 ground-dwelling mammal species, 25 bird species and 76

260 vascular plant species (Data S3).

261 We used generalized additive mixed models (GAMMs; Wood 2006) to establish relationships

262 between species' occurrence and time since fire. GAMMs are a flexible, non-parametric form of

263 regression that use smoothing functions to model non-linear relationships. Study sites were

264 spatially clustered in 100-ha landscape sampling units, so landscape and site were specified as

265 random effects to accommodate variance associated with the nestedness of the study design

266 (Zuur et al. 2009). Time since fire was specified as a non-linear smoothed term, and the degree

267 of smoothing was calculated within the model-fitting procedure (Wood 2006). We used species'

268 occurrence (presence-absence) data as the response variable because most species occurred at

269 low prevalence. Models were used to produce predicted probabilities of occurrence for each

270 species at equidistant predictor levels along the time-since-fire gradient, and probabilities of

271 occurrence were then split by growth-stage category. GAMMs were run in $\mathrm{R}$ using the package

272 gamm4 (Wood and Scheipl 2014).

273 Subsequent steps paralleled those applied to the artificial datasets (Fig. 2). First, we

274 systematically varied the total number of species included in the optimization routine (we

275 considered five scenarios: 20 species, 40, 60, 80 and 100); then we used the standardised

276 measure of Levins' index of niche breadth to measure species' growth-stage specialization

277 (Levins 1968) (Data S3). We randomly selected a specialist species per growth stage: shrubby

278 fireweed (Senecio minimus; recently burnt specialist (standardised Levins' index $\left(B_{\mathrm{A}}\right)=0.28$ );

279 sword tussock-grass (Poa ensiformis; middle specialist $\left(B_{\mathrm{A}}=0.54\right.$ (a relatively high standardised

280 Levin, but the most specialist species in the dataset)); forest starwort (Stellaria flaccida; late 
281 specialist $\left.\left(B_{\mathrm{A}}=0.17\right)\right)$. We did not identify any early specialists, and therefore tested a total of

28220 species-inclusion scenarios on the case-study dataset (five scenarios where the total number

283 of species varied $\times$ four scenarios where specialist species were excluded (including a scenario

284 where no species were excluded)).

285 Results

286 Survey-design scenarios

287 Reducing the total number of sites relative to the most exhaustive sampling scenario (200 sites)

288 resulted in a decrease in the precision of the biodiversity index, but varying the distribution of

289 sites among growth stages had little influence (Fig. 3). Patterns were consistent in communities

290 A and B; the greatest drop in the precision of the biodiversity index occurred when the total

291 number of sites was 40 or fewer, and when the number of sites in the late growth stage was

292 limited to $10 \%$ of the total. Although patterns associated with the two communities were

293 consistent, the precision of biodiversity indices associated with Community A was

294 approximately double the precision associated with Community B under all survey-design

295 scenarios (Fig. 3).

296 The survey-design scenarios had little influence on the precision and shape of the optimal growth

297 stage structure for either community when the total number of sites was 80 or more (Fig. 3,

298 Appendix S2, Fig. S1 and Fig. S2). Precision associated with the mean proportional coverage of

299 the early growth stage presented an exception; it was low relative to all other growth stages

300 under all scenarios. When the total number of sites was 20 , limiting the number of sites in the

301 late growth stage resulted in a small decrease in its proportional coverage, and consequently a

302 change in the shape of the optimal growth-stage structure. Limiting the number of sites in other

303 growth stages had a negligible influence on the shape of the optimal growth-stage structure when

304 there were 20 sites, although precision was generally poor for both communities.

305 Species-inclusion scenarios

306 For both communities, the precision of the biodiversity index and optimal growth stage structure

307 was very poor if fewer than 50 species were included, and it steadily improved as the number of

This article is protected by copyright. All rights reserved 
species increased (Fig. 4). The species-inclusion scenarios had little influence on the shape of

309 the optimal growth-stage structure for Community A (Appendix S2, Fig. S3); the mean

310 proportions of each growth stage were approximately equal irrespective of the total number of

311 species and exclusion of specialist species. However, in Community B the shape of the optimal

312 growth-stage structure differed according to the species exclusion tests (Appendix S2, Fig. S4).

313 For example, exclusion of a species that specialized on the early growth stage resulted in a

314 decrease in the proportional coverage of the early category from 0.2 to approximately zero.

315 Changes in the shape of the optimal growth-stage structure arising from the exclusion of species

316 that specialized on other growth stages were less pronounced.

317 The precision of the biodiversity index and optimal growth-stage structure associated with the

318 case-study dataset also improved markedly as the number of species included increased (Fig. 5).

319 Further, the shape of the optimal growth-stage structure changed when certain specialist species

320 were excluded (Appendix S2, Fig. S5); exclusion of a species that specialized on the middle

321 growth stage (sword tussock-grass) resulted in a reduction in the proportional coverage of both

322 the early and middle growth stages to zero. Exclusion of a recently-burnt specialist (shrubby

323 fireweed) or a late specialist (forest starwort) had a comparatively minor influence on the shape

324 of the optimal growth-stage structure.

\section{Discussion}

326 Growth-stage optimization provides an appealing basis for ecological fire management (Di

327 Stefano et al. 2013; DELWP 2015; Kelly et al. 2015), but the influences of survey design and

328 input data on results are unknown. Our results showed that the precision of the biodiversity

329 index and optimal growth stage structure for a given community increased with the number of

330 sites and the number of species included in input data, but that gains in precision lessened

331 beyond approximately 80 sites and 80 species. The distribution of sites among growth stages

332 and exclusion of specialist species were less influential provided that input data comprised

333 multiple specialists per growth stage. We discuss the insights arising from this work and the

334 implications for ecological fire management.

335 Unsurprisingly, our results showed that the precision of the biodiversity index and optimal

336 growth stage structure increased with both the total number of sites and the total number of 
337 species. Further, the precision associated with the mean proportional coverage of a particular 338 growth stage reflected the number of species that specialized on that growth stage. Our findings 339 indicate that gains in precision lessen beyond approximately 80 sites, and that fewer sites may be 340 sufficient for reasonably precise optimization results. Importantly, if the precision associated 341 with simulated optimization results is poor, the optimal growth stage structure derived from real 342 data may not represent species' "true" requirements.

343 While precision increased with the total number of sites, the distribution of sites among growth 344 stages was less important. Limiting the number of sites in a growth stage to $10 \%$ of the total had 345 little influence on optimization results except when there were only 20 sites in total, when the 346 shape of the optimal growth stage structure changed slightly. This is reassuring since it is rarely 347 possible to sample growth stages evenly because limited spatial coverage of one or more 348 categories exists at a given time. In general, survey designs comprising unevenly sampled 349 growth stages should yield robust conservation targets as long as the total number of sites is 350 adequate.

351 Similarly, incomplete sampling of species in a community is unlikely to have a major influence 352 on the shape and precision of the optimal growth stage structure unless an excluded species is the 353 only specialist on a particular growth stage. Rather than feeding biodiversity survey data directly 354 into the black box of growth-stage optimization, we recommend examining relationships 355 between individual species and time since fire as a preliminary step. If two or more specialists 356 are identified per growth stage, optimization results are likely to be fairly insensitive to the 357 addition or removal of a species from input data. We emphasize that specialization spans a 358 continuum and although the stronger the degree of specialization the greater species' influence 359 on results, our case-study data demonstrated that weaker specialists can also be influential.

360 Although exclusion of specialist species did not have a marked influence on the shape or 361 precision of the optimal growth-stage structure, we identified steady increases in precision with 362 increases in the total number of species included in input data. A high level of uncertainty 363 surrounded optimization results derived from fewer than 50 species, and precision increased 364 quite rapidly until around 80 species were included. These findings underscore the fact that the 365 growth-stage optimization approach was developed as a multispecies conservation solution and 366 will be less appropriate for restricted subsets of species. Our results support the view of Di 
367 Stefano et al. (2013) that as many species from as many taxonomic groups as possible should be 368 used in its application. Ideally, groups such as reptiles, invertebrates and fungi should be 369 incorporated, as well as soil seedbank data, which can show large differences in species 370 composition and fire responses relative to aboveground vascular plant (Wills and Read 2007;

371 Lewis et al. 2012; Di Stefano et al. 2013; Chick et al. 2016).

372 In capturing the growth-stage requirements of a wide range of species, the needs of rare species 373 may be accommodated. However, growth-stage optimization should not be used as a broad-

374 bush conservation solution in isolation of other methods (Di Stefano et al. 2013), and we 375 highlight the importance of identifying (a) highly specialist species and (b) species of 376 conservation concern. Nevertheless, the capacity of growth stage optimization to accommodate 377 data from multiple taxa presents a major advance over alternative approaches (Clarke 2008;

378 Driscoll et al. 2010; Di Stefano et al. 2013; Kelly et al. 2015).

379 In general, the influences of species-inclusion scenarios on optimization results were consistent 380 among the artificial communities and case-study data, indicating that our simulations reflected 381 reality. However, optimization results derived from communities comprising a large number of 382 species that respond to growth stage, such as faunal communities typical of forest systems in the 383 southwestern USA (e.g. Kalies et al. 2010), are likely to be relatively insensitive to species 384 inclusions. A greater degree of caution should be applied if a smaller proportion of species 385 respond to growth stage. In several southeastern Australian ecosystems, for example, a relatively 386 low proportion of animal species respond to growth stage or time since fire (Watson et al. 2012; 387 Sitters et al. 2014b; Swan et al. 2014). Moreover, species' responses can be idiosyncratic and 388 site-specific (Driscoll and Henderson 2008; Nimmo et al. 2014). The sensitivity of optimization 389 results to species inclusions could be lessened through the inclusion of a wider range of 390 taxonomic groups. If a relatively low proportion of species respond to growth stage, it is 391 essential that underlying survey data are sound because erroneous observations will generate 392 unreliable optimization results. For example, we did not conduct our analyses under scenarios of 393 differing detection probabilities, and instead assumed that species were detected when present; 394 any variation in detection probability among species and growth stages is likely to translate to 395 bias in conservation targets (e.g. MacKenzie et al. 2002). We recommend following general 396 advice regarding design of landscape-scale biodiversity surveys (Buckland et al. 2012) and in 
397 particular, survey design should allow measurement of the detection probability for each species 398 unless it is possible to count all individuals of all species at each site. Additionally, we advocate 399 use of a resampling procedure to provide a measure of the precision associated with optimization 400 results.

401 A final caveat is that robust conservation targets rely on ecologically meaningful growth-stage 402 delineations that capture major stages of vegetation growth and development after fire (Di 403 Stefano et al. 2013; Kelly et al. 2015). In cases where growth stage delineations are unknown, 404 suitable categories could be identified using a cluster analysis (McGarigal et al. 2000). The 405 appropriate number of growth stages is likely to vary among vegetation types and regions, as 406 well as taxonomic groups. For example, birds have been shown to respond to coarse 407 successional groupings comprising only two growth stages (Taylor et al. 2012; Sitters et al. 408 2014a). If different taxonomic groups in the same system respond to different numbers of 409 growth stages, we suggest using the largest number of growth stages recognized by a group.

\section{Conclusions}

411 Growth-stage optimization provides a tractable method for linking fire management objectives

412 with multispecies conservation outcomes, but the precision of optimization results depends

413 critically on choices regarding input data. Based on our analysis we recommend that survey

414 designs for precise conservation targets would ideally involve at least 80 sites, and include at 415 least 80 species. More sites and more species will yield increasingly reliable conservation 416 targets, but fewer might be adequate in some cases. The distribution of sites among growth 417 stages is less important, and exclusion of species is unlikely to have a major influence on results, 418 irrespective of their growth-stage specialization, as long as several species specialize on each 419 growth stage. Departures from an ideal survey design are likely to be less critical if a large 420 proportion of the species in a community respond to time since fire. We emphasise the 421 importance of examining responses of individual species to time since fire, and using resampling 422 to define the precision associated with conservation targets. Together, our results form a 423 reproducible guide to designing ecological surveys that yield precise conservation targets 424 through growth-stage optimization, and help to sustain biodiversity in fire-prone environments.

\section{Acknowledgements}


426 This work was funded by the Victorian State Department of Environment, Land, Water and 427 Planning. Parks Victoria and the Holsworth Wildfire Research Endowment contributed funding 428 for collection of the case-study data. We thank Fiona Christie, Garry Cheers, Peter Collins and 429 many volunteers for helping with collection of empirical data, and we are grateful to Andreas 430 Schweiger and Graeme Gange for assistance with coding.
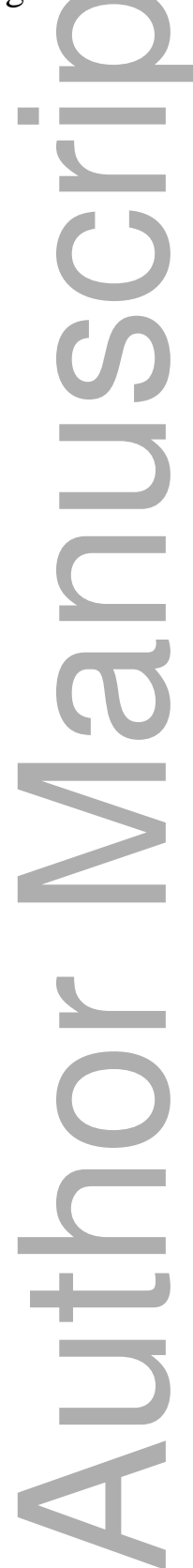


\section{Literature cited}

Bond, W. J. and J. E. Keeley. 2005. Fire as a global "herbivore": the ecology and evolution of flammable ecosystems. Trends in Ecology \& Evolution 20:387-394.

Bradstock, R. A., M. Bedward, A. M. Gill and J. S. Cohn. 2005. Which mosaic? A landscape ecological approach for evaluating interactions between fire regimes, habitat and animals. Wildlife Research 32:409-423.

Buckland, S. T., S. R. Baillie, J. M. Dick, D. A. Elston, A. E. Magurran, E. M. Scott, R. I. Smith, P. J. Somerfield, A. C. Studeny and A. Watt. 2012. How should regional biodiversity be monitored? Environmental and Ecological Statistics 19:601-626.

Buckland, S. T., A. E. Magurran, R. E. Green and R. M. Fewster. 2005. Monitoring Change in Biodiversity through Composite Indices. Philosophical Transaction: Biological Sciences 360:243-254.

Buckland, S. T., A. C. Studeny, A. E. Magurran, J. B. Illian and S. E. Newson. 2011. The geometric mean of relative abundance indices: a biodiversity measure with a difference. Ecosphere 2:art100.

Bureau of Meteorology. 2016. Climate Data Online. P. in. Bureau of Meteorolog, Canberra, Australia http://www.bom.gov.au/climate/data.

Burgess, E. E. and M. Maron. 2016. Does the response of bird assemblages to fire mosaic properties vary among spatial scales and foraging guilds? Landscape Ecology 31:687-699.

Cheal, D. 2010. Growth stages and tolerable fire intervals for Victoria's native vegetation data sets. Fire and adaptive management. P. in. Victorian Department of Sustainability and Environment, Melbourne, Australia.

Chick, M. P., J. S. Cohn, C. R. Nitschke and A. York. 2016. Lack of soil seedbank change with time since fire: relevance to seed supply after prescribed burns. International Journal of wildland Fire 25:849-860.

Clarke, M. F. 2008. Catering for the needs of fauna in fire management: science or just wishful 
thinking? Wildlife Research 35:385-394.

Cohn, J. S. S., J. Di Stefano, F. Christie, G. Cheers and A. York. 2015. How do heterogeneity in vegetation types and post-fire age-classes contribute to plant diversity at the landscape scale? Forest Ecology and Management 346:22-30.

Davies, A. B., P.Eggleton, B. J. van Rensburg and C. L. Parr. 2012. The pyrodiversitybiodiversity hypothesis: a test with savanna termite assemblages. Journal of Applied Ecology 49:422-430.

DELWP. 2015. Policy Position - Measuring ecosystem resilience in strategic bushfire management planning. Victorian Department of Environment, Land, Water and Planning. Melbourne, Australia.

Doherty, T. S., E. J. B. van Etten, R. A. Davis, C. Knuckey, J. Q. Radford and S. A. Dalgleish. 2017. Ecosystem Responses to Fire: Identifying Cross-taxa Contrasts and Complementarities to Inform Management Strategies. Ecosystems 20:1-13.

Driscoll, D. A. and M. K. Henderson. 2008. How many common reptile species are fire specialists? A replicated natural experiment highlights the predictive weakness of a fire succession model. Biological Conservation 141:460-471.

Driscoll, D. A., D. B. Lindenmayer, A. F. Bennett, M. Bode, R. A. Bradstock, G. J. Cary, M. F. Clarke, N. Dexter, R. Fensham, G. Friend, M. Gill, S. James, G. Kay, D. A. Keith, C. MacGregor, J. Russell-Smith, D. Salt, J. E. M. Watson, R. J. Williams and A. York. 2010. Fire management for biodiversity conservation: Key research questions and our capacity to answer them. Biological Conservation 143:1928-1939.

Farnsworth, L. M., D. G. Nimmo, L. T. Kelly, A. F. Bennett and M. F. Clarke. 2014. Does pyrodiversity beget alpha, beta or gamma diversity? A case study using reptiles from semiarid Australia. Diversity and Distributions 20:663-673.

Fernandes, P. M., G. M. Davies, D. Ascoli, C. Fernandez, F. Moreira, E. Rigolot, C. R. Stoof, J. A. Vega, D. Molina, C. Fernández, F. Moreira, E. Rigolot, C. R. Stoof, J. A. Vega, D. Molina, C. Fernandez, F. Moreira, E. Rigolot, C. R. Stoof, J. A. Vega, D. Molina, C. 
Fernández, F. Moreira, E. Rigolot, C. R. Stoof, J. A. Vega and D. Molina. 2013. Prescribed burning in southern Europe: Developing fire management in a dynamic landscape. Frontiers in Ecology and the Environment 11:e4-e14.

Fox, B. J. 1982. Fire and mammalian secondary succession in an Australian coastal heath. Ecology 63:1332-1341.

Fuhlendorf, S. D., D. E. Townsend, R. D. Elmore and D. M. Engle. 2010. Pyric-Herbivory to Promote Rangeland Heterogeneity: Evidence From Small Mammal Communities. Rangeland Ecology \& Management 63:670-678.

Giljohann, K. M., M. A. McCarthy, L. T. Kelly and T. J. Regan. 2015. Choice of biodiversity index drives optimal fire management decisions. Ecological Applications 25:264-277.

Hale, S., D. G. Nimmo, R. Cooke, G. Holland, S. James, M. Stevens, N. De Bondi, R. Woods, M. Castle, K. Campbell, K. Senior, S. Cassidy, R. Duffy, B. Holmes and J. G. White. 2016. Fire and climatic extremes shape mammal distributions in a fire-prone landscape. Diversity and Distributions 22:1127-1138.

Johnson, S. 2016. The NLopt nonlinear-optimization package. <http://ab-initio.mit.edu/nlopt>.

Kalies, E. L., C. L. Chambers and W. W. Covington. 2010. Wildlife responses to thinning and burning treatments in southwestern conifer forests: A meta-analysis. Forest Ecology and Management 259:333-342.

Kelly, L. T., A. F. Bennett, M. F. Clarke and M. A. McCarthy. 2015. Optimal fire histories for biodiversity conservation. Conservation Biology 29:473-481.

Kelly, L. T., D. G. Nimmo, L. M. Spence-Bailey, R. S. Taylor, S. J. Watson, M. F. Clarke and A. F. Bennett. 2012. Managing fire mosaics for small mammal conservation: a landscape perspective. Journal of Applied Ecology 49:412-421.

Levins, R. 1968. Evolution in changing environments. P. in. Princeton University Press, Princeton, NJ.

Lewis, T., M. Reif, E. Prendergast and C. Tran. 2012. The effect of long-term repeated burning 
and fire exclusion on above- and below-ground Blackbutt (Eucalyptus pilularis) forest vegetation assemblages. Austral Ecology 37:767-778.

Loh, J., R. E. Green, T. Ricketts, J. Lamoreux, M. Jenkins, V. Kapos and J. Randers. 2005. The Living Planet Index: using species population time series to track trends in biodiversity. Philosophical transactions of the Royal Society of London. Series B, Biological sciences 360:289-95.

MacKenzie, D. I., J. D. Nichols, G. B. Lachman, S. Droege, J. Andrew, C. A. Langtimm and C. A. Langtimm. 2002. Estimating Site Occupancy Rates When Detection Probabilities Are Less Than One. Ecology 83:2248-2255.

Maravalhas, J. and H. L. Vasconcelos. 2014. Revisiting the pyrodiversity-biodiversity hypothesis: long-term fire regimes and the structure of ant communities in a Neotropical savanna hotspot. Journal of Applied Ecology 51:1661-1668.

Martin, R. and D. Sapsis. 1992. Fires as agents of biodiversity: pyrodiversity promotes biodiversity. P. in Proceedings of the Symposium on Biodiversity in Northwestern California. Wildland Resources Centre, University of California, Berkeley, CA.

McCarthy, M. A. 2012. Review of resilience concepts and their measurement for fire management. Department of Sustainability and Environment, Melbourne.

McCarthy, M. A., A. L. Moore, J. Krauss, J. W. Morgan and C. F. Clements. 2014. Linking Indices for Biodiversity Monitoring to Extinction Risk Theory. Conservation Biology 28:1575-1583.

McGarigal, K., S. Cushman and S. Stafford. 2000. Multivariate Statistics for Wildlife and Ecology Research. P. in. Springer-Verlag, New York.

Nimmo, D. G., L. T. Kelly, L. M. Farnsworth, S. J. Watson and A. F. Bennett. 2014. Why do some species have geographically varying responses to fire history? Ecography 37:805813.

Parr, C. L. and A. N. Andersen. 2006. Patch mosaic burning for biodiversity conservation: a critique of the pyrodiversity paradigm. Conservation Biology 20:1610-1619. 
Penman, T. D., F. J. Christie, A. N. Andersen, R. A. Bradstock, G. J. Cary, M. K. Henderson, O. Price, C. Tran, G. M. Wardle, R. J. Williams and A. York. 2011. Prescribed burning: how can it work to conserve the things we value? International Journal of Wildland Fire 20:721733.

Pons, P. and M. Clavero. 2010. Bird responses to fire severity and time since fire in managed mountain rangelands. Animal Conservation 13:294-305.

R Core Team. 2016. R: A Language and Environment for Statistical Computing. R Foundation for Statistical Computing, Vienna, Austria.

Robinson, N. M., S. W. J. Leonard, A. F. Bennett and M. F. Clarke. 2014. Refuges for birds in fire-prone landscapes: The influence of fire severity and fire history on the distribution of forest birds. Forest Ecology and Management 318:110-121.

Schweiger, A. H., S. D. H. Irl, M. J. Steinbauer, J. Dengler and C. Beierkuhnlein. 2016. Optimizing sampling approaches along ecological gradients. Methods in Ecology and Evolution 7:463-471.

Sitters, H., F. J. Christie, J. Di Stefano, M. Swan, T. Penman, P. C. Collins and A. York. 2014a. Avian responses to the diversity and configuration of fire age classes and vegetation types across a rainfall gradient. Forest Ecology and Management 318:13-20.

Sitters, H., F. Christie, J. Di Stefano, M. Swan, P. Collins and A. York. 2014b. Associations between occupancy and habitat structure can predict avian responses to disturbance: Implications for conservation management. Forest Ecology and Management 331:227-236.

Di Stefano, J., M. A. McCarthy, A. York, T. J. Duff, J. Slingo and F. J. Christie. 2013. Defining vegetation age class distributions for multispecies conservation in fire-prone landscapes. Biological Conservation 166:111-117.

Stephens, S. L., J. D. McIver, R. E. J. Boerner, C. J. Fettig, J. B. Fontaine, B. R. Hartsough, P. L. Kennedy and D. W. Schwilk. 2012. The Effects of Forest Fuel-Reduction Treatments in the United States. Bioscience 62:549-560.

Svanberg, K. 2002. A class of globally convergent optimization methods based on conservative 
convex separable approximations. SIAM Journal on Optimization 12:555-573.

Swan, M., F. Christie, H. Sitters, A. York and J. Di Stefano. no date. Predicting faunal fire responses in heterogeneous landscapes: the role of habitat structure. Ecological Applications.

Swan, M., J. Di Stefano, F. Christie, E. Steel and A. York. 2014. Detecting mammals in heterogeneous landscapes: implications for biodiversity monitoring and management. Biodiversity and Conservation 23:343-355.

Taylor, R. S., S. J. Watson, D. G. Nimmo, L. T. Kelly, A. F. Bennett and M. F. Clarke. 2012. Landscape-scale effects of fire on bird assemblages: does pyrodiversity beget biodiversity? Diversity and Distributions 18:519-529.

Watson, S. J., R. S. Taylor, D. G. Nimmo, L. T. Kelly, A. Haslem, M. F. Clarke and A. F. Bennett. 2012. Effects of time since fire on birds: How informative are generalized fire response curves for conservation management? Ecological Applications 22:685-696.

Wickham, H. 2007. Reshaping Data with the \{reshape Package. Journal of Statistical Software $21: 1-20$.

Wickham, H. 2011. The Split-Apply-Combine Strategy for Data Analysis. Journal of Statistical Software 40:1-29.

Wickham, H. and R. Francois. 2015. dplyr: A Grammar of Data Manipulation. <https://cran.rproject.org/package $=$ dplyr $>$.

Wills, T. J. and J. Read. 2007. Soil seed bank dynamics in post-fire heathland succession in south-eastern Australia. Plant Ecology 190:1-12.

Wood, S. N. 2006. Generalized additive models. An introduction with R. P. in. Chapman and Hall, Boca Raton, Florida, USA.

Wood, S. and F. Scheipl. 2014. gamm4: Generalized additive mixed models using mgcv and lme4. <https://cran.r-project.org/package=gamm4>.

Zuur, A. F., E. N. Ieno, N. J. Walker, A. A. Saveliev and G. M. Smith. 2009. Mixed Effects

This article is protected by copyright. All rights reserved 
Models and Extensions in Ecology with R. P. in Statistics for Biology and Health. Springer, New York.

\section{Supporting Information}

Additional supporting information may be found in the online version of this article at http://onlinelibrary.wiley.com/doi/10.1002/eap.xxxx/suppinfo

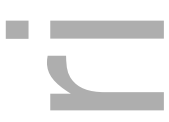

\section{Data Availability}

Data available from the Dryad Digital Repository: http://dx.doi.org/10.5061/dryad.jd523

Table 1. Frequencies of species in Communities A and B that did not respond to time since fire, or exhibited positive, negative or unimodal responses. Functions and parameter ranges corresponding to each response shape are provided. See Data S1 for a full list of species and their responses.

\begin{tabular}{lllll}
\hline & No response & Positive linear & Negative linear & Unimodal \\
\hline $\begin{array}{l}\text { Frequency in } \\
\text { Community A }\end{array}$ & 30 & 26 & 26 & 16 \\
$\begin{array}{l}\text { Frequency in } \\
\text { Community B }\end{array}$ & 66 & 16 & 8 & 10 \\
$\begin{array}{l}\text { Function } \\
\text { Range of } a\end{array}$ & $y=c$ & $y=a x+c$ & $y=a x+c$ & $y=a x^{2}+b x$ \\
& NA & $0.0012-0.0053$ & $-0.0060-$ & $-0.00130-$ \\
Range of $b$ & NA & NA & -0.0013 & -0.00009 \\
Range of $c$ & $0.12-0.94$ & $-0.22-0.49$ & NA & $0.005-0.031$ \\
Number of & 1 & 2 & 2 & NA \\
parameters & & & & 2 \\
\hline
\end{tabular}


Figure 1. Examples of relationships between the abundance of artificial species and time since fire. Gray vertical lines delineate growth-stage categories: recently burnt ( $\mathrm{R} ; 0-3$ time-since-fire units), early (E; 3-12 units), middle (M; 12-40 units), late (L; 40-100 units).

Figure 2. Workflow for testing the influence of survey-design or species-inclusion scenarios on (1) the precision of the biodiversity index, (2) the precision of the optimal growth stage structure and (3) the shape of the optimal growth stage structure.

Figure 3. The influence of survey-design scenarios (the total number of sites, and the distribution of sites among growth stages) on the precision of the biodiversity index and optimal growth stage structure for communities A and B. Precision is measured by the standard deviation of results derived from 1,000 iterations. NA indicates that sites were not limited to $10 \%$ of the total in any growth stage, and numbers of sites per growth stage were equal.

Figure 4. The influence of species-inclusion scenarios (the total number of species, and the exclusion of specialist species) on the precision of the biodiversity index and optimal growth stage structure for communities A and B. Precision is measured by the standard deviation of results derived from 10,000 iterations (100 resamples per 100 species subsets).

Figure 5. The influence of species-inclusion scenarios (the total number of species, and the exclusion of specialist species) on the precision of the biodiversity index and optimal growth stage structure for ground-dwelling mammals, birds and vascular plants in forby forest. Precision is measured by the standard deviation of results derived from 10,000 iterations (100 resamples per 100 species subsets).

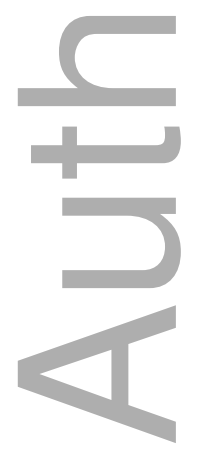

This article is protected by copyright. All rights reserved 


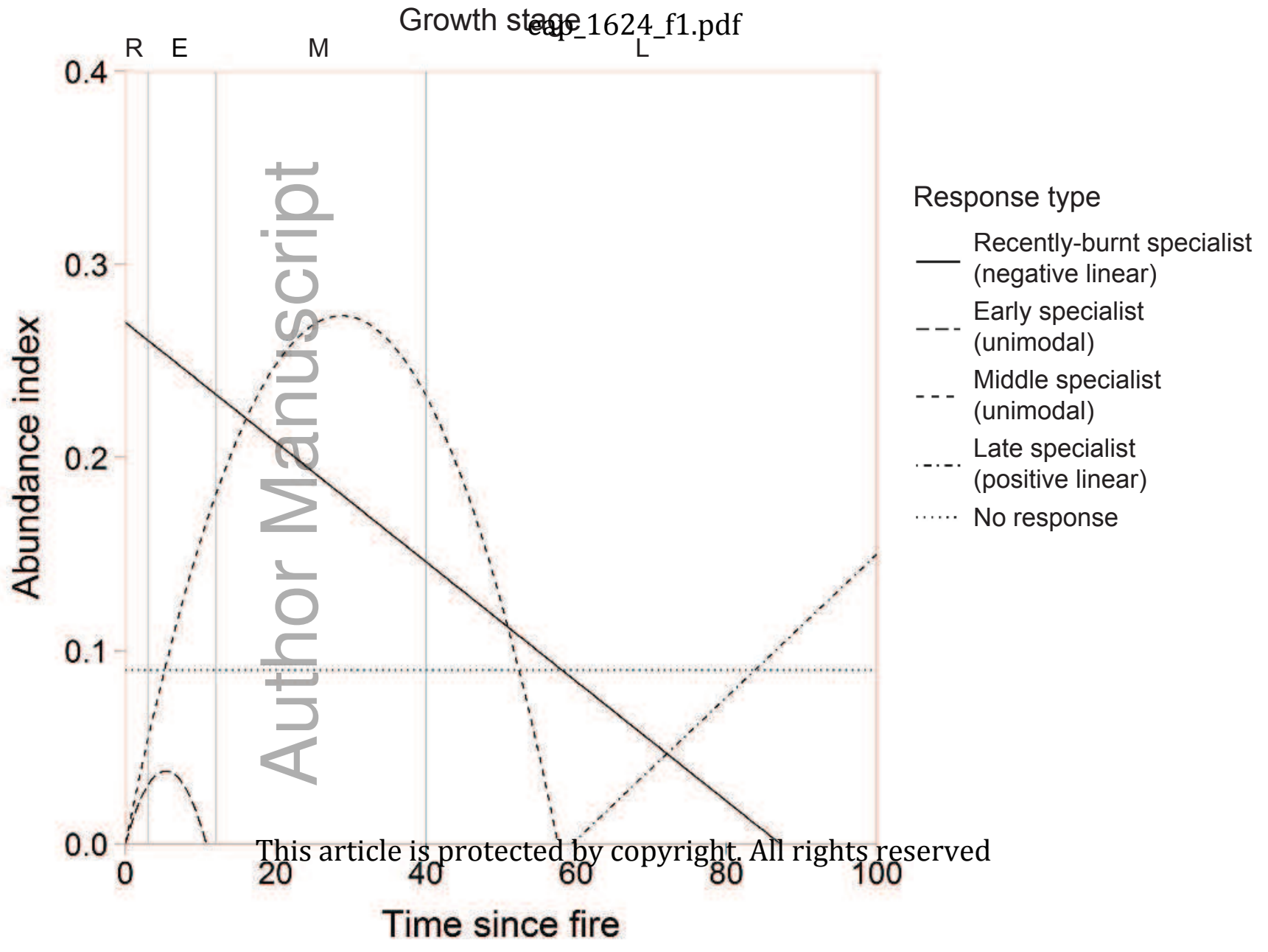




\section{eapes624 f2" edffaset}

50 observations per species $(n=100)$ per growth stage $(n=4)$

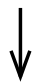

Define scenario of interest and subsample<smiles>CC(C)(C)C</smiles>

Resample with replacement

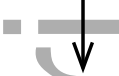

Calculate mean abundance per species per growth stage<smiles>CC1(c2ccccc2)CC1</smiles>

Conduct growth stage optimisation on resampled dataset<smiles>C=CC</smiles>

Measure precision of the:

- biodiversity index (standard deviation of 1000 values)

- optimal growth stage structure (mean of standard deviations corresponding to each growth stage)

Determine shape of the optimal growth stage structure by plotting mean proportions and confidence intervals

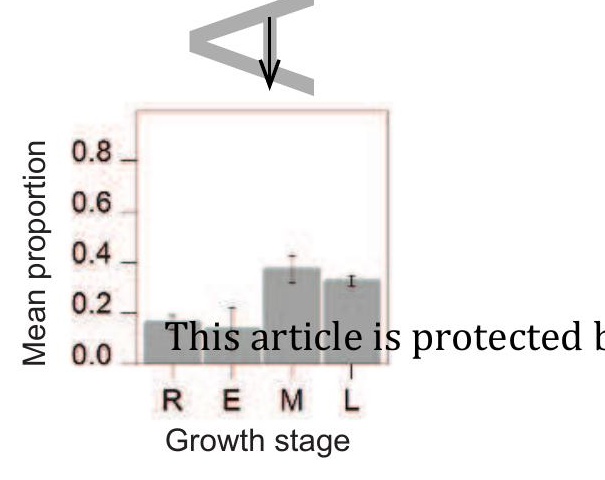




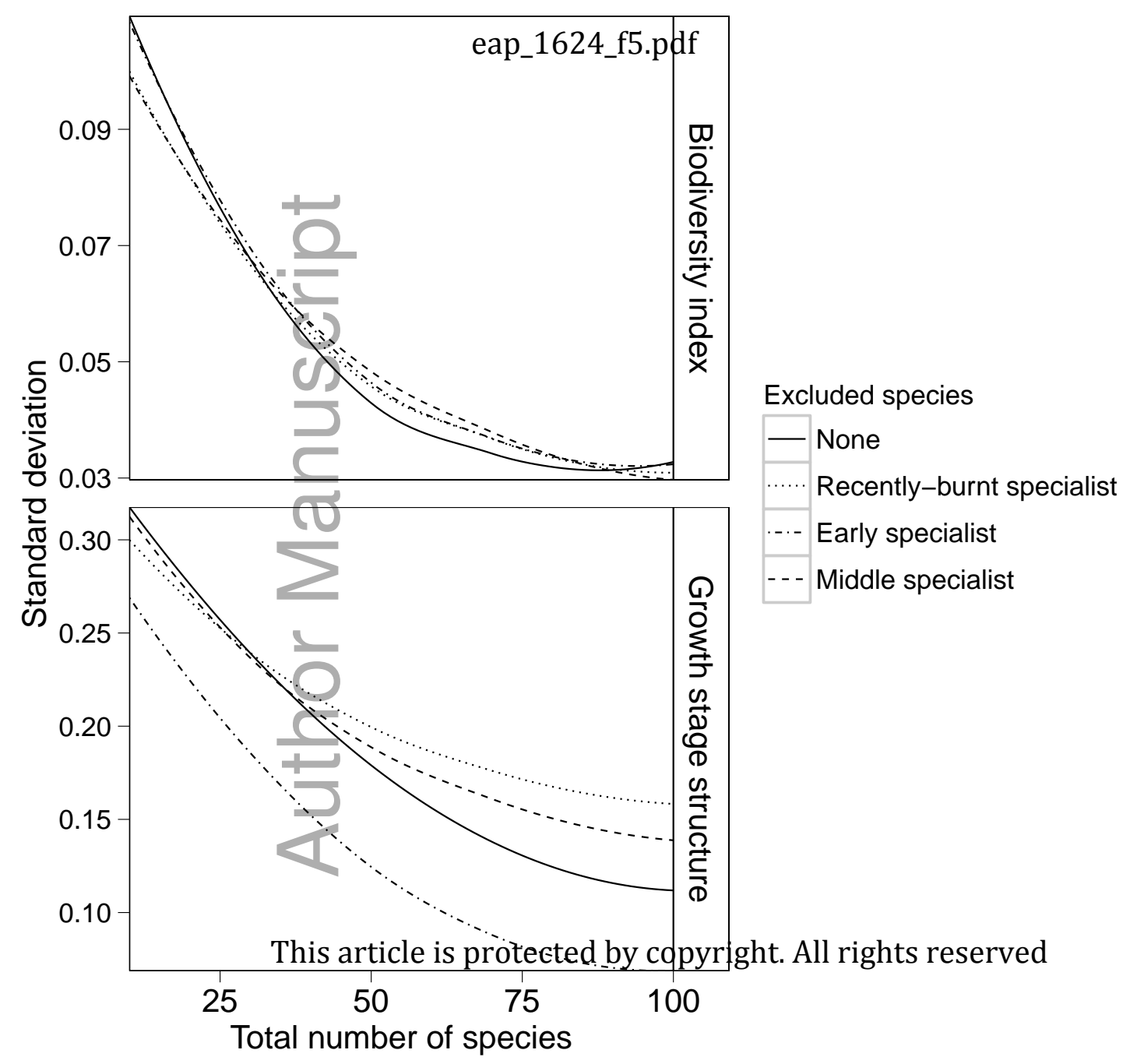




\section{University Library}

\section{- M M N E R VA A gateway to Melbourne's research publications}

Minerva Access is the Institutional Repository of The University of Melbourne

Author/s:

Sitters, H;Di Stefano, J;Wills, T;Swan, M;York, A

Title:

Survey design for precise fire management conservation targets

Date:

2018-01-01

Citation:

Sitters, H., Di Stefano, J., Wills, T., Swan, M. \& York, A. (2018). Survey design for precise fire management conservation targets. ECOLOGICAL APPLICATIONS, 28 (1), pp.35-45. https:// doi.org/10.1002/eap.1624.

Persistent Link:

http://hdl.handle.net/11343/294008 